\title{
Collapse Turbulence in Nonlinear Schrödinger Equation
}

\author{
Yeo-Jin Chung*, Pavel M. Lushnikov ${ }^{\dagger}$ and Natalia Vladimirova ${ }^{\dagger}$ \\ ${ }^{*}$ Department of Mathematics, Southern Methodist University Dallas, TX 75275, USA \\ ${ }^{\dagger}$ Department of Mathematics and Statistics, University of New Mexico, Albuquerque, NM 87131, USA
}

\begin{abstract}
We consider a nonlinear Schrödinger equation (NLS) with dissipation and forcing in critical dimension. Without both linear and nonlinear dissipation NLS results in a finite-time singularity (collapse) for any initial conditions. Dissipation ensures collapse regularization. If dissipation is small then multiple near-singular collapses are randomly distributed in space and time forming collapse turbulence. Collapses are responsible for non-Gaussian tails in the probability distribution function of amplitude fluctuations which makes turbulence strong. Power law of non-Gaussian tails is obtained for strong NLS turbulence.
\end{abstract}

Keywords: Strong turbulence, collapse, regularization of singularity, intermittency

PACS: $47.27 .-\mathrm{i} 42.65 . \mathrm{Jx} 52.38 . \mathrm{Hb}$

The nonlinear Schrödinger equation (NLS) is one of the most widespread and universal model in nonlinear science. NLS describes e.g. a propagation of light in media with Kerr-type nonlinearity [1] and in plasma [2], an evolution of Bose-Einstein condensate [3,4], and a propagation of wave packet in a very general class of nonlinear media [5]. NLS for a complex wave amplitude $\psi$ in space dimension $D$ is given by

$$
i \psi_{t}+\nabla^{2} \psi+|\psi|^{2 \sigma} \psi=0, \quad \sigma>0,
$$

where $\sigma$ determines the order of nonlinear interaction. Most common in applications is $\sigma=1$.

Solutions of Eq. (1) exist globally for $\sigma D<2$ and NLS is called subcritical. In particular, one-dimensional cubic NLS ( $D=1$ and $\sigma=1$ ) is integrable by the inverse scattering transform [6]. For $\sigma D \geq 2$ the amplitude of solution can reach infinity in a finite time, i.e. a blow up occurs. The blow up is accompanied by a dramatic contraction of the spatial extent, $L(t)$, of function $\psi$, that is called collapse [7]. A sufficient condition for the collapse in that case is $H<0$, where

$$
H=\int\left(|\nabla \psi|^{2}-\frac{1}{\sigma+1}|\psi|^{2 \sigma+2}\right) d \mathbf{r}
$$

is the Hamiltonian (the energy functional) and Eq. (1) can be rewritten in the Hamiltonian form as

$$
i \psi_{t}=\frac{\delta H}{\delta \psi^{*}}
$$

Besides the Hamiltonian, another conserved integral of Eq. (1) is

$$
N=\int|\psi|^{2} d \mathbf{r}
$$

which is usually refer to as either number of particles in quantum mechanical applications, or optical power in nonlinear optics applications.

We consider critical dimension $\sigma D=2$, which is the border of collapse existence and corresponds to the critical nonlinear Schrödinger equation,

$$
i \psi_{t}+\nabla^{2} \psi+|\psi|^{4 / D} \psi=0
$$

where a finite amount of the number of particles, $\lim _{t \rightarrow t_{0}} N_{\text {collapse }} \sim 1$, is captured into the collapsing region. Here $t_{0}$ is the time of singularity formation.

Near $t=t_{0}$ collapse should be regularized because the critical NLS (5) looses its applicability near singularity point. We consider the following regularized NLS (RNLS) :

$$
i \psi_{t}+(1-i a \varepsilon) \nabla^{2} \psi+(1+i \varepsilon)|\psi|^{4 / D} \psi=i \varepsilon \phi
$$



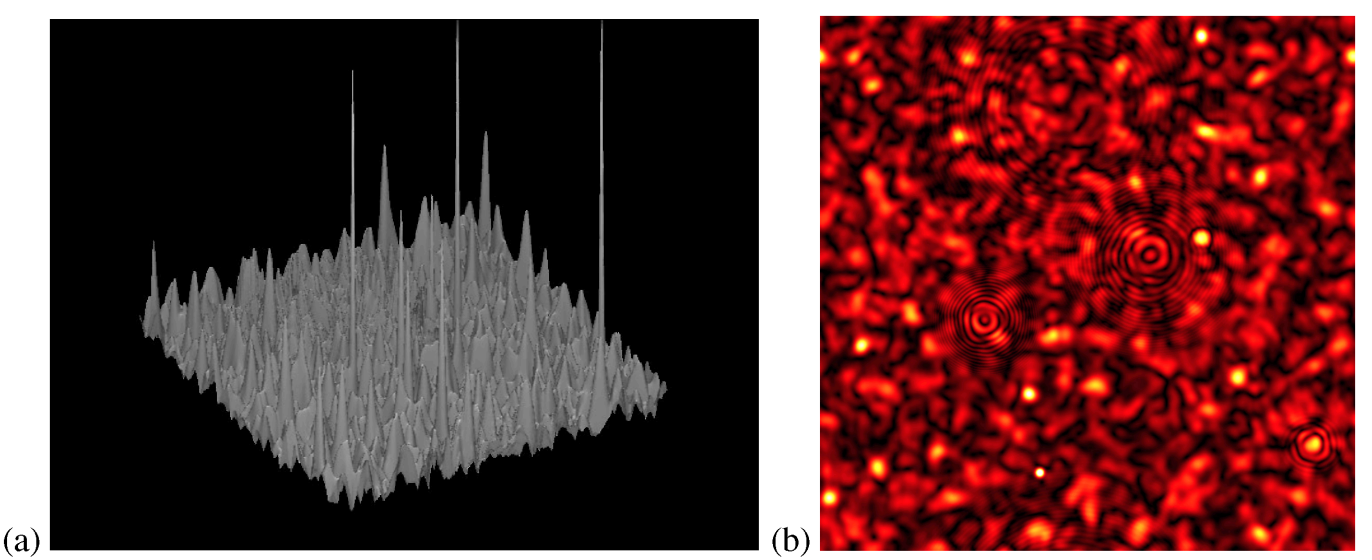

FIGURE 1. Simulation of 2D critical NLS (6) with the deterministic forcing (7), $\varepsilon=0.01$ and $b=20$. (a) Snapshot of $|\psi|$ vs. spatial coordinates $(x, y)$. (b) Snapshot of density plot for $|\psi|$ shows collapse decay with emission of cylindrical waves. Spatial grid $4096 \times 4096$ was used.

which can be also called a complex Ginzburg-Landau equation. Here $0<\varepsilon \ll 1$ is the small parameter so that to leading approximation, the critical NLS (5) is still valid. The coefficient $a \sim 1$ determines a linear dissipation and term $i \varepsilon|\psi|^{4 / D} \psi$ is responsible for nonlinear dissipation.RNLS (6) describes e.g. a propagation of light in a ring cavity with Kerr nonlinearity (see e.g. [8]) or any quite general propagation of waves in nonlinear media with complex dispersion and nonlinear dissipation [5]. The right hand side (rhs) of Eq. (6) provides forcing and depends on the specific physical model. We consider two types of forcing. First is a deterministic forcing

$$
\phi=b \psi, \quad b \sim 1,
$$

which corresponds to a linear instability in a system. Second type is a random additive forcing

$$
\phi=\xi(t, \mathbf{r}),
$$

where zero in average stochastic term $\xi$ is a random Gaussian variable with

$$
\left\langle\xi\left(t_{1}, \mathbf{r}_{1}\right) \xi^{*}\left(t_{2}, \mathbf{r}_{2}\right)\right\rangle=\delta\left(t_{1}-t_{2}\right) \chi\left(\left|\mathbf{r}_{1}-\mathbf{r}_{2}\right|\right),
$$

$\chi\left(\left|\mathbf{r}_{1}-\mathbf{r}_{2}\right|\right)=b_{g} \exp \left[\left|\mathbf{r}_{1}-\mathbf{r}_{2}\right|^{2} /\left(2 l_{c}^{2}\right)\right], l_{c}$ is the correlation length of pump, and $b_{g}$ is the normalization constant.

The both types of forcing (7) and (8) pump the number of particles into the system at large spatial scales, which leads to modulation instability. Development of modulation instability results in generation of spatial harmonics with a typical wavenumber $k_{\max } \simeq(2 / D)^{1 / 2}\left|\psi_{0}\right|^{2 / D}$, where $\left|\psi_{0}\right|$ is the typical amplitude of $\psi$. Nonlinear stage of modulation instability development results in multiple collapses which are randomly distributed in space and time as shown in Figure 1 for $D=2$.

Dissipation becomes important in RNLS (6) only near maximum amplitude of each collapse, when dissipation stops temporal growth of collapse amplitude and causes subsequent decay of collapse event into outgoing cylindrical waves as in Figure 1b. Most dissipation occurs shortly after collapse event when these short wavelength almost linear waves dissipate due to linear dissipative term in (6). Fast decay of the number of particles $N$ after each collapse collapse event can be seen in simulations of one dimensional (1D) collapse turbulence in Figure 2. We also observe that for $\varepsilon \ll 1$ system behavior depends only weakly on type of forcing (7) and (8). Level of forcing determines average amplitude $\left|\psi_{0}\right|$ but otherwise both (7) and (8) give similar results for probability distribution function (PDF) of amplitude fluctuations. Thus RNLS (6) shows a universal collapse turbulence picture with pumping of the number of particles at large scales and dissipation at small scales. First studies of collapse turbulence of similar types were performed in Refs. [9] (for $D=1$ ) and [10] (for $D=2$ ). RNLS also shows dissipative anomaly because the number of particles dissipated in each collapse event $\triangle N \sim 1$ (see e.g. Figure 2) and does not depend on $\varepsilon$ while if we set $\varepsilon=0$ then RNLS (6) turns in NLS (5) with singular collapses.

We study here tails of PDF $\mathscr{P}$ of amplitude $|\psi|$. Figures $3 \mathrm{a}$ and $3 \mathrm{~b}$ show PDF $\mathscr{P}(h)$ for absolute values $|\psi|=h$ obtained from a direct numerical simulation of Eq. (6) for $D=1$ with the deterministic forcing from Eq. (7) (Figure 

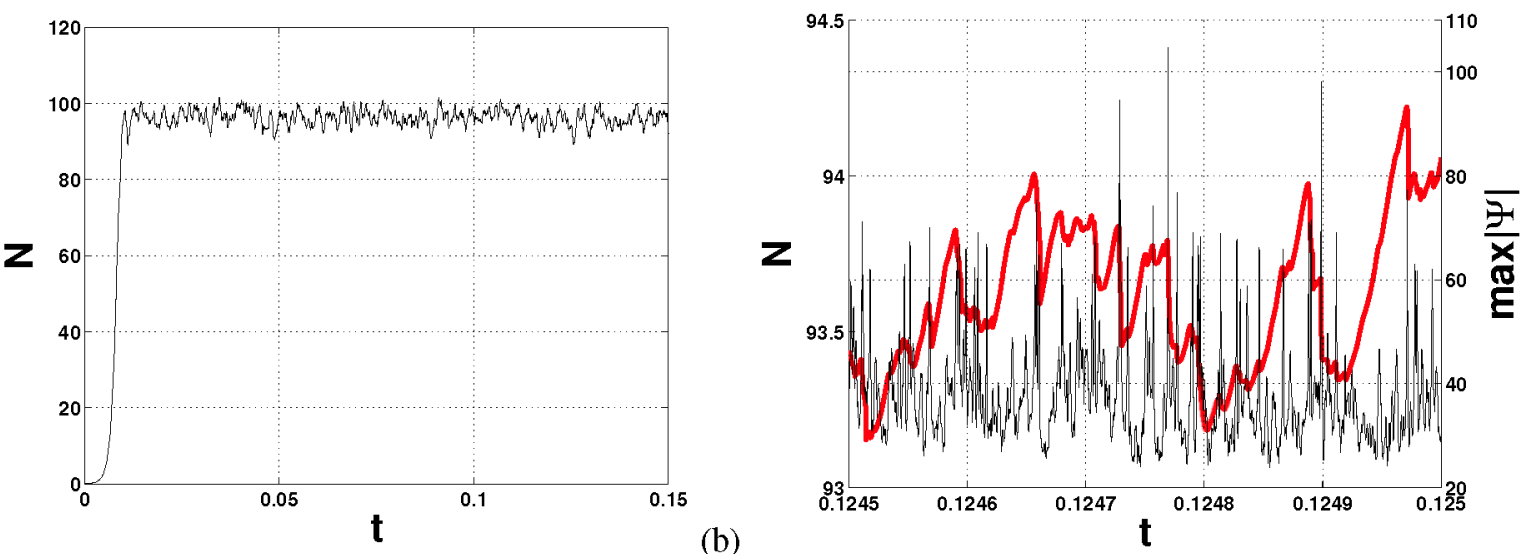

(a)

FIGURE 2. Time dependence of the number of particles $N$ for numerical simulation of 1D critical NLS (6) with $\varepsilon=10^{-3}$ and the deterministic forcing (7) with $b=2 \cdot 10^{5}$. (a) Initially $N$ grows until statistical steady-state is achieved so that the average value of $N$ remain constant. (b) Zoom-in at the smaller scale in $t$. Thick red curve (solid thick curve in black-and-white) corresponds to $N(t)$. Solid black thin curve gives time dependence of maximal spatial value of $|\psi|$. Scale for $N$ is on the left vertical axis and scale for $\max |\psi|$ is on the right vertical axis, respectively. It is seen that sharp decreases of $N$ is due to dissipation from large collapses. These decreases are compensated in average by a growth of $N$ from the linear forcing.

(a)

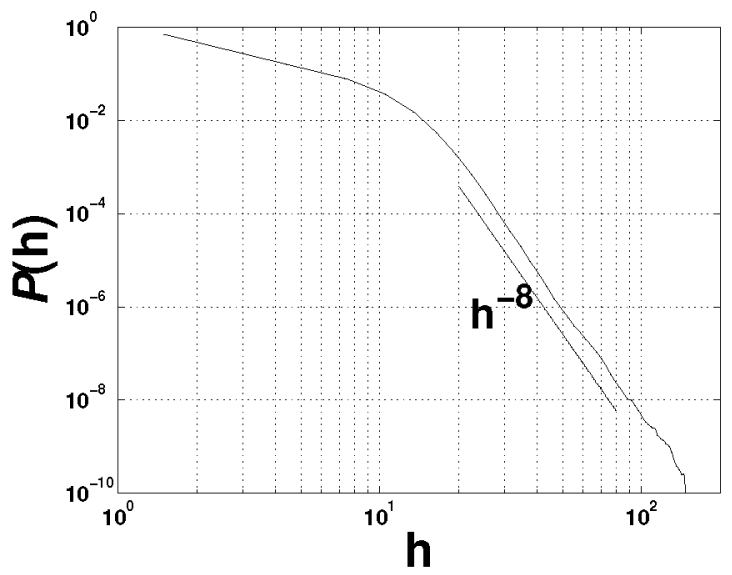

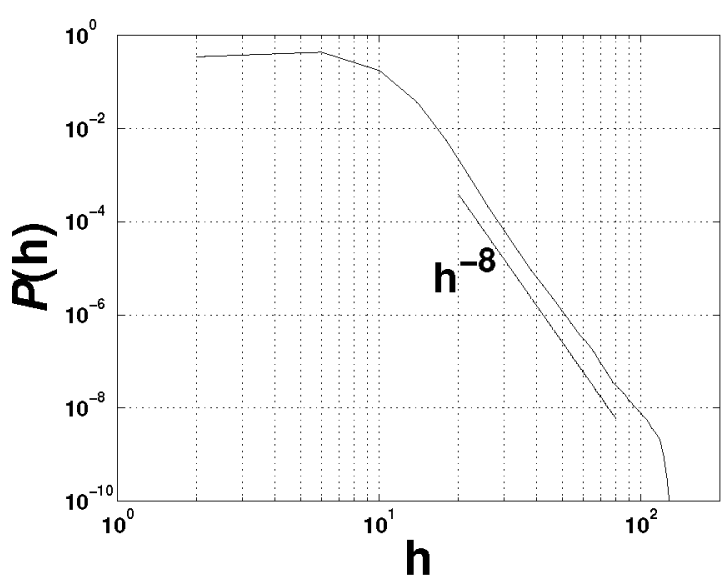

FIGURE 3. Log-log plot of $\operatorname{PDF} \mathscr{P}(h)$ for $h=|\psi|$ values obtained from a direct numerical simulation of Eq. (6) for $\varepsilon=10^{-3}$ with (a) the deterministic forcing (7) and (b) the stochastic forcing (8) (b). In both cases the tails of $\mathscr{P}(h)$ matches well to $h^{-8}$ law except very large values of $h \sim 100$ where dissipation strongly suppress collapse growth. For smaller $\varepsilon$ that saturation occurs for larger $h$.

3a) and the stochastic forcing from Eq. (8) (Figure 3b). Assuming that collapses are distributed uniformly in space and time, PDF $\mathscr{P}$ is determined numerically as integral over computational domain, $\mathscr{P}(h)=\frac{\int \delta(|\boldsymbol{\psi}(\mathbf{r}, t)|-h) d \mathbf{r} d t}{\int d \mathbf{r} d t}$. It is seen that in both cases the tails of PDF matches $h^{-8}$ law,

$$
\mathscr{P}(h) \propto h^{-8} \quad \text { for } \quad h \gg 1 .
$$

Similar scaling for deterministic force was obtained for a first time in Ref. [9].

Figure 4 shows results of 2D simulations which gives the following tails of PDF in 2D:

$$
\mathscr{P}(h) \propto h^{-6} \quad \text { for } h \gg 1 .
$$




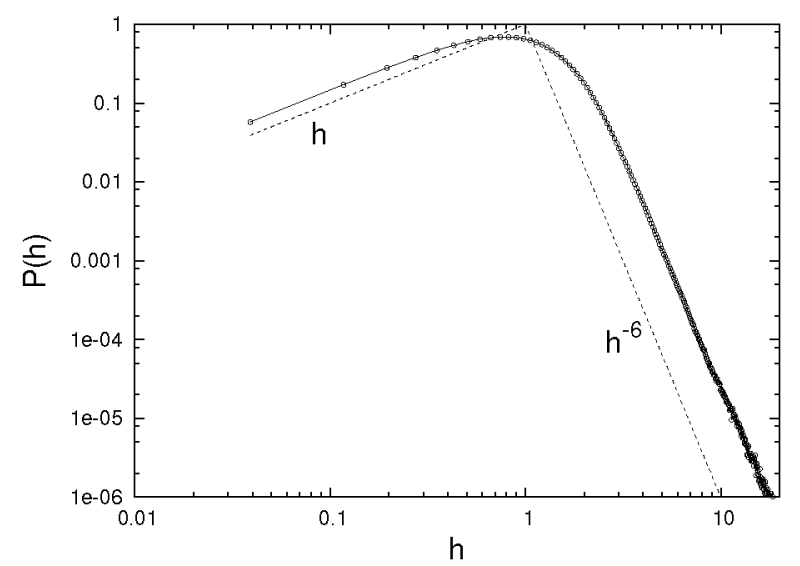

FIGURE 4. Log-log plot for PDF $\mathscr{P}(h)$ in 2D. Parameters are the same as in Figure 1.

There are two major contribution to PDF $\mathscr{P}$. First contribution comes from individual collapse events. Self-similar solution of NLS (5) near collapse is well approximated by the following radially-symmetric solution [1]:

$$
\begin{array}{r}
|\psi(r, t)|=\frac{1}{L^{D / 2}} R(\rho), \\
\rho=\frac{r}{L},
\end{array}
$$

where $L(t)$ determines a time-dependent spatial scale of collapse and $R$ is the ground state soliton solution of NLS (5) given by

$$
\nabla^{2} R_{0}-R_{0}+R_{0}^{4 / D+1}=0
$$

This first contribution gives $\mathscr{P}\left(h \mid h_{\max }\right) \simeq \frac{c}{h^{3+4 / D}} \Theta\left(h_{\max }-h\right)$, where $h_{\max }$ is the maximum amplitude of a given collapse event, where $\Theta(x)$ is the Heaviside step function. Second contribution is determined by the PDF $\mathscr{P}_{\max }\left(h_{\max }\right) \propto$ $1 / h_{\max }^{2}$ for $h_{\max }$. Combining both contributions together we obtain:

$$
\mathscr{P}(h)=\int \mathscr{P}\left(h \mid h_{\max }\right) \mathscr{P}_{\max }\left(h_{\max }\right) d h_{\max } \simeq \int \frac{c}{h^{3+4 / D}} \Theta\left(h_{\max }-h\right) \mathscr{P}_{\max }\left(h_{\max }\right) d h_{\max }=\frac{c}{h^{4+4 / D}},
$$

which is our main result. This power tail of PDF $\mathscr{P}$ corresponds to strongly non-Gaussian fluctuations, i.e. intermittency of turbulence. Compare (15) with both (10) and (11) we conclude that they both match to (15). Another example of strong turbulence dominated by near singular event is turbulence in forced Burgers equation where a tail of PDF for negative gradients has well-known $(-7 / 2)$ power law [11].

\section{REFERENCES}

1. C. Sulem, and P.L. Sulem. Nonlinear Schrödinger Equations: Self-Focusing and Wave Collapse (World Scientific, 1999).

2. S.L. Musher, A.M. Rubenchik, and V.E. Zakharov, Phys. Rep. 252177 (1995).

3. M.H. Anderson et al., Science 269, 198 (1995).

4. F. Dalfovoet et al., Rev. Mod. Phys. 71, 463 (1999).

5. V.E. Zakharov, V.S. Lvov, and G. Falkovich, Kolmogorov Spectra of Turbulence I: Wave turbulence (Springer-Verlag, New York, 1992)

6. V.E. Zakharov and A.B. Shabat, Sov. Phys. JETP 34, 62 (1972).

7. E.A. Kuznetsov, and V.E. Zakharov (Eds.) Wave Collapse (World Scientific Publishing Company, 2007).

8. P.M. Lushnikov, and M. Saffman, Phys. Rev. E, 62, 5793 (2000).

9. H. Iwasaki, S. Toh, Progr.Theor.Phys.87, 1127 (1992).

10. S. Dyachenko, A.C. Newell, A.Pushkarev, V.E. Zakharov, Physica D 57, 96-160 (1992).

11. W. E, K. Khanin, A. Mazel, Y. Sinai, "Probability Distribution Functions for the Random Forced Burgers Equation", Phys. Rev. Lett. 78, 1904-1907 (1997). 